\title{
"UT ILLI NON REPUGNET ESSE IN MATERIA" La dottrina di Zaccaria Pasqualigo (1600-1664) sulla natura della metafisica e del suo oggetto
}

Marco Forlivesi*

RESUMO - Zacarias Pasqualigo desenvolveu sua doutrina concernente à natureza da metafísica e ao seu objeto nos anos vinte do século XVII. Ela pertence, no meio católico, ao grupo de reação às teses propostas por Francisco Suárez sobre esse assunto. Pasqualigo desenvolve uma metafísica cujo objeto formal não é o ente transcendental, mas o ente considerado como um modo de ser da quidditas rei omnino abstrahens a materia. Contudo, o 'prescindindo da matéria' que é próprio a esse ente não é entendido por Pasqualigo como uma indiferença ao que é material e ao que é espiritual. Ao contrário, de acordo com esse autor, o ente do qual a metafísica trata é intrinsecamente material. Não obstante, a metafísica trata do ente material considerado separado da matéria puramente por meios da ação da mente. Como resultado, de acordo com Pasqualigo, a metafísica é uma ciência superior à física, mas não é uma ciência superior à teologia racional; com efeito, a metafísica pasqualiguiana é quase completamente separada de qualquer tipo de teologia.

PALAVRAS-CHAVE - Zacarias Pasqualigo (1600-1664). História da Metafísica. História da Epistemologia. Objeto da Metafísica. Escopo da Metafísica.
ABSTRACT - Zaccaria Pasqualigo developed his doctrine concerning the nature of metaphysics and of its object in the twenties of the 17th century. It belongs to the group of reactions, in the Catholic milieu, to the theses propounded by Francisco Suárez on this topic. Pasqualigo develops a metaphysics whose formal object is not the transcendental being, but the being considered as the way of being of the quidditas rei omnino abstrahens a materia. However, the 'prescinding from matter' that is proper of this being is not understood by Pasqualigo as an indifference to what is material and to what is spiritual. On the contrary, according to this author the being that metaphysics deals with is intrinsically material. Therefore, metaphysics deals with material being considered apart from matter purely by means of an action of the mind. As a result, according to Pasqualigo metaphysics is a science superior to physics, but it is not a science superior to rational theology; actually, Pasqualighian metaphysics is almost completely separated from whatever kind of theology.

KEY WORDS - Zaccaria Pasqualigo (16001664). History of Metaphysics. History of Epistemology. Subject of Metaphysics. Scope of Metaphysics.

* Dottore di Ricerca. Collaboratore di Ricerca. Dipartimento di Filosofia dell'Università degli Studi di Padova.

\begin{tabular}{|l|l|l|l|l|l|}
\hline VERITAS & Porto Alegre & v. 54 & n. 3 & set./dez. 2009 & p. 156-172 \\
\hline
\end{tabular}




\section{Introduzione: alcune reazioni alla dottrina suareziana sulla natura della metafisica nel primo quarto del XVII secolo}

Alla fine del XVI secolo Francisco Suárez aveva sviluppato una concezione della metafisica come scienza unitaria ma asimmetrica delle rationes generalissime e degli enti spirituali. Il gesuita spagnolo riteneva che la trattazione dell'ente trascendentale e quella dell'ente spirituale fossero inseparabili e che entrambe fossero di competenza della metafisica. Egli riteneva anche che il grado di analiticità con cui la metafisica studia l'ente spirituale fosse, almeno in qualche misura, maggiore del grado di analiticità con cui tale scienza studia l'ente materiale. Infine riteneva che l'oggetto adeguato della metafisica - così come l'oggetto di qualsiasi scienza - ricapitolasse precisamente i rapporti che legano i diversi oggetti e le diverse parti di questa scienza. Suárez esprimeva quest'ultima tesi scrivendo che oggetto adeguato della metafisica è l'ente in quanto ente reale: non un puro contenuto concettuale, né l'ente trascendentale preso senza qualificazioni, bensì l'ente trascendentale preso nel suo esser astratto dalla materia secundum esse et considerationem e, pertanto, in quanto includente alcuni inferiori e non altri. ${ }^{1}$

Con questa proposta si confrontano tutti gli autori della prima metà del XVII secolo, sia di ambito cattolico che di ambito protestante. In ambito protestante, tuttavia, essa o non è colta nella sua specificità, oppure - in particolare in ambito calvinista - è radicalmente respinta, a favore dell'antitetica proposta di Benet Perera. ${ }^{2}$ In ambito cattolico le reazioni sono più complesse. In effetti, la dottrina di Suárez sulla natura della metafisica e del suo oggetto costituisce una soluzione elegante ad alcuni dei problemi sollevati su questo tema tra la metà del XIII e la fine del XVI secolo. ${ }^{3}$ Ciò non fa sì, tuttavia, che essa sia inattaccabile.

Un primo punto contestabile risiede nel modo in cui il gesuita granadino intende l'unità di una scienza. Suárez concepisce la scienza come un insieme di conoscenze stabili unificato dall'unità di ciò che quella scienza considera. Nel caso della metafisica questa concezione si traduce nella tesi per cui l'oggetto adeguato di tale scienza è l'ente considerato nel suo essere astratto secundum esse et considerationem. La scienza, però, può essere concepita in un modo differente, ossia come la concepivano i nominales: come un complesso di proposizioni, ciascuna vista come una scienza 'semplice'. Questa concezione della scienza

1 Circa la dottrina di Suárez, mi permetto di rinviare ad alcuni miei saggi: FORLIVESI. 2004a. Precedente versione su supporto cartaceo: ID. 2004b. Versione abbreviata in lingua inglese: ID. 2005.

2 Cfr. LAMANNA. 2009, e POZZO. 2009.

3 Per una breve ricapitolazione della storia del problema dell'oggetto della metafisica tra medioevo ed età moderna, cfr. FORLIVESI. 2009a, e PORRO. 2005, p. XIX-XX. 
non sembra immediatamente conciliabile con quella del Granadino. Il criterio unificatore della scienza, infatti, va in questo caso cercato sul piano sintattico e consiste in quel termine al quale sono riconducibili tutti i termini presenti nelle proposizioni di quella scienza presi nell'uso che di essi viene fatto. Suárez non contesta esplicitamente questa tesi; in un breve passaggio si limita a negare che vi siano tante scienze quante specie di cose. ${ }^{4}$ Al contrario, il gesuita Pedro Hurtado de Mendoza sviluppa le proprie posizioni anche a partire dall'esigenza di studiare la scienza in quanto insieme coordinato di proposizioni. ${ }^{5}$

Un secondo punto critico concerne la possibilità del darsi di un'unica scienza teoretica atta a studiare tutti gli enti reali, siano essi materiali o immateriali. Suárez esclude che vi possa essere un'unica scienza teoretica umana comprendente tutte le conoscenze particolari: ciò perché, egli argomenta, sia la matematica che la fisica possiedono principî propri, grazie ai quali esse procedono formando proposizioni evidenti e acquisendo una conoscenza dei propri oggetti più perfetta di quella acquisibile con la metafisica. Ciononostante, ci si può chiedere se sia possibile che si dia un'unica scienza superiore alle sole metafisica e fisica; una scienza distinta dalla matematica e tale da non sopprimere le differenze tra metafisica e fisica, e tuttavia tale da comprendere queste ultime come propri casi particolari. Suárez non prende in considerazione questa specifica ipotesi e non elabora argomenti contro di essa. Reputo che ciò accada perché egli ritiene di aver mostrato a sufficienza la diversità delle tre scienze speculative; nondimeno ci si può chiedere se metafisica e fisica pur essendo diverse siano abbastanza simili da poter essere comprese in un'unica scienza teoretica, così come - secondo Suárez - la scienza dell'ente astratto per indifferenza dalla materia e la scienza dell'ente astratto per esclusione positiva della materia sono simili e connesse quanto basta da dover essere comprese in un'unica scienza (ossia nella metafisica). Su questa possibilità si inserisce la speculazione del chierico regolare minore Raffaele Aversa. ${ }^{6}$

4 In effetti, si potrebbe pensare che il Granadino tenti in qualche modo di integrare questa concezione nella propria: egli sostiene, infatti, che nel soggetto conoscente ogni scienza unitaria è costituita da una pluralità di habitus correlati secondo un ordine di subordinazione.

5 Sulla concezione hurtadiana della metafisica cfr. RUFFINENGO. 2009.

6 Non vi sono studi editi sulla concezione della fisica e della metafisica proposta da Aversa. Il testo nel quale egli espone il proprio pensiero su questo tema è la Philosophia metaphysicam physicamque complectens quaestionibus contexta, pubblicato a Roma in due tomi rispettivamente nel 1625 e 1627 e riedito, come 'seconda edizione', a Bologna nel 1650. Mi permetto di rinviare il lettore alle breve disamina che ho condotto su alcuni passaggi di questo testo in FORLIVESI. 1999 (liberamente accessibile all'indirizzo internet http:// web.tiscali.it/ marcoforlivesi/ mf1999om.pdf). 
Una terza questione concerne la 'scomponibilità' dell'oggetto adeguato della metafisica. Il problema che genera questa difficoltà è noto anche a Suárez: il soggetto di una scienza dev'essere tale da far sì che di esso si dimostrino proprietà; perché ciò sia possibile, occorre che vi siano una o più rationes che fungano da termini medi in dimostrazioni in cui si dimostra che certe proprietà appartengono a tale soggetto; ne viene che il soggetto di una scienza deve possedere tale tipo di rationes. Di fronte a questo problema Suárez aveva risposto che l'ente, in quanto oggetto conoscibile, ha una ratio formale sufficiente e principî sufficienti a far sì che di esso si possano dimostrare proprietà. La soluzione del pensatore spagnolo, però, presta il fianco a una possibile critica: si potrebbe sostenere che le rationes tramite cui si dimostrano proprietà dell'ente non possono essere la ratio stessa dell'ente; al contrario, esse devono essere rationes interne all'ente e in una certa misura distinte da esso; ma se così è, l'ente che è oggetto della metafisica dev'essere concepito come composito. Su questa convinzione costruisce la propria posizione il teatino veronese Zaccaria Pasqualigo.

\section{La dottrina di Zaccaria Pasqualigo}

La produzione libraria matura di Pasqualigo ${ }^{7}$ (in due casi inserita nello Index librorum prohibitorum, con la formula donec corrigatur) è dedicata principalmente a temi di teologia morale e di diritto canonico. Qui ci interessa un lavoro che egli compose e pubblicò in età relativamente giovanile: i due ponderosi volumi di Disputationes metaphysicae. Diversamente da quanto accade nel caso di altri autori del suo tempo e similmente a quanto aveva fatto Suárez, quest'opera non è un commento alla Metafisica di Aristotele. Peraltro, essa neppure fa parte di un cursus philosophicus, non essendo né preceduta, né seguita da altri lavori di argomento filosofico. Conseguenza di ciò è che non tutto quello di cui in essa si tratta è di argomento metafisico; infatti, al fine di garantire coerenza e compiutezza al proprio discorso, Pasqualigo vi discute anche alcuni temi di logica e di fisica.

Dal punto di vista bibliologico, l'opera è costituita da due tomi, le cui pagine sono numerate autonomamente. Al frontespizio dichiarano come luogo di stampa Roma e come anno di stampa rispettivamente il 1634 e il 1636. Le approbationes di stampa datate del primo tomo sono del

7 Non mi sono noti studi storici moderni su questo autore. Fatto inspiegabile per più di una ragione, ma se non altro perché egli fu uno dei tre teologi cui nel 1633 il Sant'Uffizio fece esaminare il Dialogo sopra i massimi sistemi di Galilei al fine di stabilire se, pubblicando tale opera, l'autore avesse violato i termini di quanto impostogli nel 1616. Rinvio pertanto a VEZZOSI. 1780, p. 156-161. 
1 febbraio 1629 e del 2 luglio 1633; quelle del secondo tomo sono del 7 agosto 1632 e del 4 settembre 1633. Dal punto di vista dell'organizzazione del contenuto, l'opera è costituita da due partes, coincidenti con i due tomi, divise a loro volta in disputationes. Le disputationes delle due partes sono numerate autonomamente e sono raggruppate in insiemi ciascuno dei quali è preceduto da un titolo generale; questi raggruppamenti, tuttavia, non danno luogo a soluzioni di continuità nella numerazione delle disputationes. ${ }^{8}$

Pasqualigo già conosce e discute le tesi di Raffaele Aversa. Nella settima disputatio, dedicata al problema Quomodo philosophia speculativa dividenda sit, il nostro teatino distingue la posizione del chierico regolare minore da quella attribuita da Suárez ad Antonio Bernardi della Mirandola e a Egidio Romano. Questi, secondo il Granadino, includono nell'unica scienza speculativa anche la matematica; ${ }^{9}$ al contrario, Aversa accorpa in un'unica scienza solo metafisica e fisica. ${ }^{10}$ Nonostante questa diversità, Pasqualigo respinge l'una e l'altra tesi: se è vero, egli argomenta, che fisica e metafisica sono scienze prossime e in continuità, è anche vero che non sono tanto unite da non poterle distinguere. ${ }^{11}$

L'opposizione di Pasqualigo ad Aversa non impedisce a quest'ultimo di lasciare una traccia nella speculazione del primo. Nelle Disputationes metaphysicae del teatino, le disputationes raggruppate sotto il titolo

8 ZACHARIA Pasqualigus. Disputationes metaphysicae. 2 voll.: vol. 1, Romae: Ex typographia Andreae Phaei, 1634; vol. 2, Romae: Typis Francisci Caballi, 1636.

9 Circa la posizione di Egidio Romano, mi permetto di rinviare a FORLIVESI. 2009a. Circa la posizione di Antonio Bernardi, mi permetto di rinviare a FORLIVESI. 2004a, e ID. 2009b (si vedano, in particolare, i contributi di Marinheiro, Muccillo e Forlivesi).

10 ZACHARIA Pasqualigus. Disp. met., pars 1, De natura et divisione philosophiae, disp. 7: Quomodo philosophia speculativa dividenda sit, sect. 1: Quid sentiant auctores, n. 2-4, vol. 1, p. 40a-b.

11 ZACHARIA Pasqualigus. Disp. met., pars 1, De natura et divisione philosophiae, disp. 7, sect. $2-4$, vol. 1, p. 41a-48b. Si veda, ad esempio, ivi, sect. 2: Posterior sententia ut vera declaratur, n. 3, p. 41b-42a: "Philosophiam naturalem speculativam non esse ita unam, ut non sit divisibilis in alias partes, quae constituant scientias diversas speculativas; unde non solum scientiae simpliciter multiplicandae sunt, sed etiam scientiae speculativae. Atque principalis ratio pro hoc dicto desumitur ex diversis rationibus formalibus; in rebus enim, quae speculativo modo cognoscibiles sunt, reperiuntur plures rationes formales obiectivae, nam alia est ratio formalis entis, ut ens est; alia ratio formalis corporis, qua corpus est, alia ratio formalis dimensionis, qua dimensio est, quod adeo evidens est, ut alia probatione non egeat. Ergo debent concedi etiam diversae scientiae, quemadmodum conceduntur diversae rationes formales obiecti. Haec consequentia declaratur, scientia enim inclinat intellectum in obiectum, cum quadam adaequatione, et proportione, ita ut intellectus habeat quamdam adaequatam repraesentationem, qua sibi obiectum cognoscibile innotescat, ergo intellectus prout adaequatur cum una ratione formali obiectiva, non potest adaequari cum alia, et consequenter quot erunt rationes formales obiectivae, tot etiam erunt scientiae". 
complessivo 'De natura et divisione philosophiae' precedono quelle raggruppate sotto il titolo complessivo 'De natura et obiecto metaphysicae'. Con ciò, il teatino veronese si pone - come Aversa - tra quegli autori che determinano l'oggetto della metafisica solamente dopo aver preso in esame alcune delle caratteristiche di tale scienza. I passi che Pasqualigo compie, tuttavia, sono in qualche misura invertiti rispetto a quelli compiuti da Aversa. Le caratteristiche prese in esame da entrambi gli autori sono due: quelle che si potrebbero chiamare (sono parole mie, non di Aversa o di Pasqualigo) l'estensione e l'intensione della scienza in esame. Con 'estensione' intendo la regione della realtà di cui quella scienza di occupa, il punto più alto al quale essa giunge sulla scala che va dalle rationes più particolari a quelle più generali. Con 'intensione' intendo il grado di penetrazione di tale scienza, il grado di dettaglio con cui essa - posta la sua estensione - indaga la realtà o, in altri termini, quale porzione della scala che va dalle rationes più generali a quelle più particolari essa - a partire dal punto più alto cui essa giunge discende e abbraccia. Ora, nell'opera di Aversa la discussione rivolta a determinare l'intensione della scienza speculativa che incorpora fisica e metafisica precede la discussione rivolta a determinare l'oggetto di quella 'super'-scienza; la discussione rivolta a determinare la natura di questo oggetto è contestuale a quella rivolta a determinare l'estensione di tale scienza. Nell'opera di Pasqualigo viene innanzi tutto discussa e difesa la distinzione delle tre scienze speculative; una discussione che è contestuale a quella rivolta a determinare il grado di estensione della metafisica. A questa discussione segue quella rivolta a determinare l'intensione della metafisica, la quale a sua volta in parte precede la discussione rivolta a determinare l'oggetto di questa scienza, in parte è contestuale a essa.

Giunto alla quindicesima disputatio, il nostro autore pone il problema della determinazione della natura della metafisica in termini di determinazione dello obiectum di tale scienza. Qui egli affronta la questione nei termini di una determinazione dell'estensione di tale scienza. Pasqualigo esclude innanzi tutto che l'oggetto della metafisica comprenda l'ente di ragione e l'ente occasionale (per accidens). Esclude anche che tale oggetto sia Dio, o la sostanza immateriale in generale, o la sostanza in generale. Sostiene, invece, che esso è l'ente reale. ${ }^{12}$ La conclusione sembrerebbe del tutto tradizionale; un avvertimento dello stesso Pasqualigo, tuttavia ci fa capire fin d'ora che così non è:

12 ZACHARIA Pasqualigus. Disp. met., pars 1, De natura et obiecto metaphysicae, disp. 15: Quodnam sit obiectum adaequatum metaphysicae, vol. 1, p. 91b114b. 
cosa si debba intendere per ente reale, egli scrive, lo si dirà più avanti. ${ }^{13}$

La disputatio successiva offre una delimitazione della nozione di 'ente reale che è oggetto della metafisica' a fronte della questione dell'intensione di questa scienza. Qui Pasqualigo si pone il problema seguente. La metafisica tratta ordinariamente dell'ente, delle sue proprietà, della sostanza e dell'accidente. Ora, se diciamo che la metafisica tratta solo dell'ente in generale, allora non tratta della sostanza e dell'accidente; se diciamo che tratta dell'ente in particolare, allora tratta di tutto. ${ }^{14}$

Suárez aveva risolto il problema scrivendo che da un lato la metafisica considera l'ente anche come qualcosa che in un certo modo include i suoi inferiori; dall'altro che tale scienza si occupa solo di ciò che cade sotto il grado di astrazione che è proprio del suo oggetto. Pasqualigo fornisce una risposta assai simile. Il grado di astrazione, egli scrive, non è la ratio formale specificativa di una scienza; nondimeno, è criterio sufficiente a circoscriverla. Ora, la metafisica considera ciò che è astratto da ogni materia; pertanto comprende non solo la ratio di ente, bensì tutte quelle rationes che non implicano alcuna materia. ${ }^{15}$

13 ZACHARIA Pasqualigus. Disp. met., pars 1, De natura et obiecto metaphysicae, disp. 15, sect. 6: Statuitur obiectum metaphysicae, n. 1, vol. 1, p. 108a-b: "cum communi sententia statuendum est, obiectum metaphysicae esse ipsum ens reale. Quid autem nomine entis intelligi debeat, et quomodo assignanda sit ratio formalis, et materialis obiecti, infra declarabimus; non enim hoc commode exponi potest, nisi prius declaremus, quam late pateat, et ad quae se extendat obiectum metaphysicae, quod disp. sequenti praestabimus, deinde coarctato obiecto intra suos limites, facile declarabimus, quid nomine entis importetur, quando dicitur esse obiectum metaphysicae, et in ipso rationem materialem, atque formalem distinguemus".

14 ZACHARIA Pasqualigus. Disp. met., pars 1, De natura et obiecto metaphysicae, disp. 16: An ens, quod est obiectum metaphysicae, includat inferiora, sect. 1: Ratio dubitandi et auctorum sententiae, n. 1, vol. 1, p. 115a: "Ratio autem dubitandi est, quia, si dicimus, ens, quod est obiectum metaphysicae, non includere inferiora, iam non aget de substantia, et accidenti. Praeterea inferiora, etiam ut inferiora sunt, important rationem entis; debent ergo contineri sub obiecto metaphysicae. E contra vero, si dicamus, considerare etiam inferiora, tunc res omnes spectabunt ad obiectum metaphysicae, etiam ipsa corpora naturalia, nec multiplicandae erunt diversae scientiae".

15 ZACHARIA Pasqualigus. Disp. met., pars 1, De natura et obiecto metaphysicae, disp. 16, sect. 2: Vera sententia, vol. 1, p. 116a-118b. Si veda ivi, n. 2-3, p. 116a-b: "Ut autem certi limites pro obiecto metaphysicae constituantur, supponendum est, dari diversas abstractiones a materia, ut supra etiam tradimus. Ex eo enim, quod intellectus noster per suam vim praecisivam potest in eadem entitate diversas rationes formales distinguere, sequitur quod faciat diversas praecisiones, dum concipit unam rationem formalem sine altera. [...] Hae igitur abstractiones assignantur scientiis unicuique sua peculiaris, et licet non sint rationes formales specificativae scientiarum, sunt tamen aptae, ut coarctent, et circumscribant ea, quae ab aliqua scientia considerantur, non enim quaelibet ratio obiectiva formalis contineri potest intra quamlibet abstractionem [...]". Si veda anche ivi, 
La risposta di Pasqualigo, dicevo, è simile a quella fornita da Suárez; nondimeno non è identica. Il teatino veronese in primo luogo introduce una distinzione più netta, rispetto a quella suareziana, tra il criterio in base al quale una scienza viene circoscritta e il criterio grazie al quale una scienza viene specificata. In secondo luogo intende il criterio del 'astratto secundum esse' in modo profondamente diverso da come lo intende il gesuita spagnolo. Di questo secondo argomento dirò tra breve. Ora seguiamo Paqualigo nella determinazione della ratio dell'oggetto della metafisica.

Si è visto cosa egli scrive a proposito dell'oggetto della metafisica a fronte del tema dell'estensione e di quello dell'intensione di tale scienza. Nella disputatio seguente, la diciassettesima, egli affronta il problema a fronte del fatto che è necessario che l'oggetto di una scienza possieda cause e principî. La questione non era per nulla nuova e la risposta quasi scontata che a essa gli autori davano era che l'ente possedesse le cause e i principî richiesti. Su quali fossero queste cause e questi principî, tuttavia, non vi era unanimità. Una minoranza di scrittori, tra i quali Tommaso d'Aquino, Antonio Andrés, Domenico di Fiandra e Francisco de Araujo, sostenevano che l'ente (o, più precisamente, quella ratio che è l'ente che è soggetto della metafisica) avesse vere cause; tutti gli altri ammettevano che esso avesse cause e principî solo in senso lato.

Pasqualigo risponde al problema sviluppando una soluzione radicale e inedita. L'oggetto di una scienza - egli scrive - deve, per essere tale, avere cause intrinseche e principî intrinseci; ma l'ente comunissimo, trascendentale, non ha nulla di tutto questo; dunque non può essere l'oggetto della metafisica. Se la metafisica è una scienza, dunque, essa deve avere per oggetto qualcosa di completo (ossia dotato di cause e principî intrinseci); viceversa, essa può essere una scienza precisamente grazie al fatto che ha un oggetto siffatto. ${ }^{16}$

n. 4, p. 117a: "Supposito ergo, quod supra docuimus, metaphysicae correspondere abstractionem ab omni materia. Dicendum est, Metaphysicam non considerare omnes inferiores rationes quidditativas rerum, sed illas tantum, quae continentur intra abstractionem metaphysicam, seu quae nihil materiale involvunt. Unde non considerat omnia inferiora entis secundum quamcumque rationem, sed solum secundum aliquas. [...] Ecce ergo, quomodo non solam rationem entis speculandam concedit metaphysicae, sed etiam alias rationes; et quomodo non omnes rationes quidditativas inferiorum illi assignat; sed relinquit aliquas coeteris scientiis".

16 ZACHARIA Pasqualigus. Disp. met., pars 1, De natura et obiecto metaphysicae, disp. 17: Quodnam ens fit obiectum metaphysicae; ubi de ratione formali et materiali obiectiva, sect. 1-2, vol. 1, p. 121a-128b. Si veda ad esempio ivi, sect. 1: Ens communissime sumptum non esse illud, quod est obiectum metaphysicae, n. 3, p. 121b; n. 13, p. 125a e n. 14, p. 125b: "Ens communissime, et abstractissime sumptum, prout est prima ratio transcendentalis, et fundamentum totius realitatis, non esse illud ens, quod constituendum est pro obiecto metaphysicae. Atque ut validam rationem, quam demonstrativam existimo pro praesenti, materia. 
Cos'è, allora, l'oggetto della metafisica? È, risponde il nostro autore, l'ente che è costituito dalle sue cause intrinseche e dai suoi principî intrinseci. Per l'esattezza, la ratio materiale dell'oggetto della metafisica è la quidditas rei abstrahens omnino a materia, ove per quidditas - precisa ulteriormente il nostro autore - si intende tutto ciò che è effetto dell'unità dei suoi principî quidditativi. Dunque, la ratio formale dell'oggetto della metafisica è il particolare modo d'essere della quidditas astratta dalla materia; tale modo d'essere, spiega Pasqualigo, è normalmente detto 'ente'. Questa precisazione permette di dire, conclude il nostro autore, che l'ente reale che è oggetto della metafisica porta con sé i suoi principî quidditativi, che non sono altro che le sue parti metafisiche; in sintesi, esso è la quidditas comprensiva dei suoi principi. ${ }^{17}$

Con ciò le sorprese per il lettore non sono finite. Occorre ancora vedere cosa esattamente intenda Pasqualigo con l'espressione 'del tutto astratto dalla materia' e di quale quidditas parli. La questione emerge nelle due disputationes seguenti, dedicate rispettivamente al posto della trattazione di Dio e a quello della trattazione delle sostanze separate in

afferamus. Praemittendum est, quod obiectum cuiuscunque scientiae debet habere suas causas, atque principia intrinseca, quibus tanquam ex elementis coalescat, atque quale est obiectum, talia etiam debent esse principia sic obiectum physicum debet habere principia physica, obiectum vero metaphysicum, sufficiet, si habeat principia metaphysica, quae nos secundum rationem in ipso distinguamus. [...] Ens [...] est simplicissimum conceptus obiectivus non resolubilis in plures conceptus, ergo nullo pacto potest habere principia intrinseca, sive ea per modum abstracti, et concreti explicentur, sive per modum formae, aut quocunque alio modo. Consequentia ista probatur, nam si haberet principia intrinseca, vel essent entia, vel non. Non potest dici quod sint, quia idem esset principium, et principiatum, et rursus illa principia, cum sint entia deberent, habere principia entis [...]; si vero non sunt entia, quomodo, quod est nihil, potest esse principium reale entis? [...] Constat ergo, quod ens communissime sumptum nequeat esse obiectum metaphysicae".

17 ZACHARIA Pasqualigus. Disp. met., pars 1, De natura et obiecto metaphysicae, disp. 17, sect. 3-4, vol. 1, p. 128b-133b. Si veda ad esempio ivi, sect. 3: Statuitur propria sententia, n. 7, p. 130a-b: "cum enim quidditas abstrahens ab omni materia acquirat peculiarem modum essendi, acquirit etiam peculiarem modum scibilitatis, etenim scibilitas consequitur ad esse rei, atque illi proportionatur. Haec igitur scibilitas est ratio formalis obiectiva metaphysicae, quae principaliter reperitur in quidditate completa, quam diximus esse obiectum metaphysicae, et suo modo, atque cum quadam proportione in principiis, et passionibus talis quidditatis completae, quatenus scilicet passiones habent commensurationem cum quidditate completa, atque principia concurrunt, ut quo ad eius constitutionem. Si autem quaeratur, quidnam sit haec ratio formalis; quae importat talem scibilitatem. Respondendum est. Esse peculiarem illum modum essendi, quae habet res ex eo, quod abstrahit ab omni materia; sicuti enim res completa immersa in materia ex vi sui modi essendi habet rationem naturae, ita quidditas abstrahens a materia habet suum peculiarem modum essendi, quo mediante est tali pacto speculabilis; qui nomine entis solet explicari, utpote quia est primus modus secundum naturam habendi esse, et proinde cum quadam antonomasia dicitur ens, hoc est habens esse $[\ldots] "$. 
metafisica. Nella disputatio diciottesima il nostro autore si chiede An Deus contineatur sub obiecto metaphysicae. Nella disputatio quindicesima Pasqualigo si era limitato a sostenere che né Dio, né la sostanza separata in generale sono l'oggetto della metafisica. Ora aggiunge - contestando Hurtado de Mendoza - che non solo Dio non è oggetto adeguato della metafisica, ma non vi è neppure contenuto come oggetto parziale, o di attribuzione, o in qualunque altro modo. Detto in altri termini, il teatino veronese sostiene che è preferibile che la trattazione di Dio sia riservata alla teologia soprannaturale, anche quando si considera Dio come prima causa o come ultimo fine. ${ }^{18}$

Di questa tesi Pasqualigo fornisce cinque prove. In primo luogo, sostiene, Dio non è ente; dunque non partecipa alla ragione formale dell'oggetto della metafisica. Che Dio non sia ente è dimostrabile nel modo seguente. La ratio entis in Dio è impartecipata e tale per essenza; ma la ratio entis è inclusa in ogni creatura; ne viene che se la ratio entis di Dio fosse la medesima che è inclusa nella creatura, allora ogni creatura sarebbe ente in modo impartecipato e per essenza; dunque sarebbe Dio. Inoltre: Dio è privo di principî intrinseci; ma la metafisica studia l'ente che è costituito dai suoi principî; dunque non studia l'esser ente di Dio. In secondo luogo, prosegue il teatino veronese, Dio astrae dalla materia ex natura sua e come qualcosa di singolare; invece l'oggetto della metafisica astrae dalla materia per mezzo dell'intelletto, ossia per mezzo di un'astrazione eseguita dall'intelletto (facta per intellectum); dunque Dio non ricade nell'oggetto della metafisica. In terzo luogo Dio è singolare; ma la metafisica si occupa dell'universale; dunque non si occupa di Dio. In quarto luogo i principî mediante i quali conosciamo Dio spettano alla scienza di Dio, ossia alla teologia soprannaturale; dunque non spettano alla metafisica. Infine Dio secondo la sua natura non è naturalmente conoscibile; ma la metafisica, come ogni scienza, si occupa della natura delle cose per quanto sono conoscibili naturalmente; dunque non si occupa di Dio. ${ }^{19}$

18 Cosa che egli fa nell'opera ZACHARIA Pasqualigus. Sacra speculativa doctrina de Deo coeterisque divinitus revelatis ex theologicis principijs ad scholasticae lecturae methodum deducta. Venetiis: apud Bertanos, 1650.

19 ZACHARIA Pasqualigus. Disp. met., pars 1, De natura et obiecto metaphysicae, disp. 18: An Deus contineatur sub obiecto metaphysicae, vol. 1, p. 133b-145b, in particolare sect. 3: Propria sententia, vol. 1, p. 137b-143b. Si veda ad esempio ivi, n. 1-4, vol. 1, p. 137a-b: "His adnotatis, statuimus, Deum non contineri sub obiecto metaphysicae, tanquam obiectum partiale, sive attributionis, sive quocunque alio modo. Quod enim non sit obiectum totale, iam supra ostensum fuit. Sed ad summum considerari posse a metaphysica tanquam causam, et principium extrinsecum sui obiecti; et tanquam ultimum finem: verum de facto, quia adest theologia, melius 
La natura dell'astrazione di ciò di cui la metafisica si occupa è al centro anche della disputatio diciannovesima. Qui Pasqualigo esclude anche le sostanze immateriali create dall'ambito dell'oggetto della metafisica. La prospettiva generale del nostro autore è la seguente: le sostanze immateriali prese come tali sono astratte dalla materia secundum esse, cioè per propria natura; ma la metafisica si occupa solo di rationes universaliores, cioè di rationes la cui astrazione dalla materia è effetto di un'operazione dell'intelletto; dunque la metafisica non si occupa delle sostanze immateriali.

Al centro dell'attenzione di Pasqualigo vi è, ovviamente, la premessa minore del ragionamento ora veduto. L'oggetto della metafisica, argomenta, è tratto dalla materia; dunque non gli deve ripugnare l'essere nella materia. In secondo luogo, essere astratto dalla materia solo secondo la considerazione ed essere astratto dalla materia per quel che si è (secundum rem) sono modi di astrazione opposti; dunque non possono convenire a una stessa scienza. In terzo luogo, astrarre è separare qualcosa da qualcos'altro al quale era unito; ma gli angeli non sono uniti alla materia; dunque, a rigori, non sono né astratti dalla materia, né astraibili. In quarto luogo, quando l'ente è contratto al corporeo cessa di essere oggetto della metafisica; altrettanto accade quando è contratto all'incorporeo. Infine, è un fatto che gli angeli siano meglio conosciuti in teologia; dunque è quella la scienza che se ne occupa. D'altronde, le intelligenze sono connesse alle cose sensibili solo in quanto esse sono motori dei cieli; ma tale connessione è solo contingente, dato che quel moto potrebbe provenire

ab ipsa considerari etiam secundum rationem primae causae, et ultimi finis; quia meliori modo hac cognitio potest haberi in theologia, quam in metaphysica; et deinde quia etiam ratio primae causae, et ultimi finis spectant ad obiectum proprium theologiae. Philosophi autem, quia carebant theologia, et naturaliter attingebant Deum tanquam primam causam, et ultimum finem de ipso secundum has rationes egerunt in metaphysica, tanquam in loco magis proportionato, ut desumitur ex d. Thoma [...]. Atque hoc verum esse existimo non solum iuxta nostra principia, qui posuimus obiectum metaphysicae esse quidditatem completam abstrahentem a materia, sed etiam iuxta communem opinionem, quae asserit, ens communissime sumptum esse obiectum. Unde argumentor primo. Deus non participat rationem obiectivam formalem obiecti metaphysici, ergo non est obiectum partiale metaphysicae. Consequentiam evidentem existimo [...]. [...] Quod vero spectat ad antecedens: clarum fiet infra ubi disp. 29. sect. 6. ostendemus, quod Deus non est ens, sed aliquid supereminentius, et consequenter omnes illas rationes, in quibus videtur cum creaturis saltem analogice convenire non esse tales, sed aliquid aliud supereminentialis, et alterius omnino rationis, ita ut nullam habeat convenientiam, cum creaturis". 
direttamente da Dio; dunque l'esistenza dei motori dei cieli è solo probabile. ${ }^{20}$

In definitiva, Pasqualigo sostiene che la contrazione dell'ente all'immateriale dà luogo a una scienza diversa dalla metafisica tanto quanto lo dà la contrazione dell'ente al corporeo. Si noti, tuttavia, la peculiarità della posizione del Veronese rispetto alle posizioni, ad esempio, di scotisti quali Francesco di Appignano (della Marca), Nicola Bonet o - in modo diverso - Filippo Fabri. ${ }^{21}$ La tesi secondo la quale la metafisica non si occupa delle sostanze immateriali non è sostenuta da Pasqualigo solamente sulla base della tesi per cui la metafisica tratta solo di ciò che è massimamente comune. Piuttosto, la tesi secondo la quale la metafisica non si occupa delle sostanze immateriali è sostenuta dal nostro autore sulla base della seguente considerazione: la metafisica tratta solamente di rationes astraibili dagli enti materiali e alle quali, pertanto, non ripugni di darsi in enti materiali; dunque non può occuparsi di enti spirituali.

\section{Considerazioni conclusive}

Già negli anni '80 del secolo scorso il pensiero di Zaccaria Pasqualigo era stato oggetto delle indagini di Piero Di Vona. Lo studioso napoletano

20 ZACHARIA Pasqualigus. Disp. met., pars 1, De natura et obiecto metaphysicae, disp. 19: An intelligentiae contineantur sub obiecto metaphysicae; ubi etiam quam late pateat abstractio metaphysica, vol. 1, p. 145b-153b, in particolare sect. 1: Intelligentias non claudi in obiecto metaphysicae, vol. 1, p. 145b-150b. Si veda ad esempio ivi, n. 3-4, p. 146a-b: "[...] praesens difficultas [...] procedit de intelligentiis, quatenus tales sunt, nempe substantiae quaedam spirituales per se subsistentes, et completae, ita ut non veniant in compositionem alterius, et quatenus secundum rem abstrahunt ab omni materia. Et sic [lege: si] hoc pacto considerentur existimo dicendum esse, non pertinere ad metaphysicam tanquam obiectum partiale. Et possumus argumentari primo pro hac nostra sententia ratione illa, qua etiam disp. praecedenti usui sumus, ut excluderemus Deum ab obiecto metaphysicae. Obiectum metaphysicae, sive ponatur ens, sive quidditas completa constans suis principiis intrinsecis, si consideretur ut totale, et adaequatum est, ita abstrahit a materia, ut illi non repugnet esse in materia secundum illud esse, quo abstrahit, ergo quodlibet obiectum partiale illius debet ita abstrahere ab omni materia, ut illi non repugnet esse in materia, substantiis autem spiritualibus, quales sunt angeli, omnino repugnat esse in materia, ergo nequeunt esse obiectum partiale metaphysicae".

21 È possibile che Fabri e Pasqualigo si conoscessero, giacché Pasqualigo esercitò la docenza di filosofia nel collegio dei teatini di Padova mentre Fabri era docente di teologia in via Scoti presso l'Università di quella città. Sta però di fatto che nessuno dei due cita l'altro nelle sue opere. Si può in effetti osservare che Fabri scrisse la propria opera di metafisica prima che le Disputationes metaphysicae di Pasqualigo fossero pubblicate (Fabri morì nel 1630) e, viceversa, Pasqualigo pubblicò la propria opera di metafisica prima che fosse pubblicata, postuma, quella di Fabri (le cui Expositiones et disputationes in XII libros Aristotelis Metaphysicorum furono pubblicate nel 1637). 
aveva richiamato l'attenzione su temi in parte differenti da quello preso in esame nel presente studio; tra questi, la tesi del Veronese circa l'attribuibilità a Dio del termine 'ente'. Pasqualigo, osservava Di Vona, si chiede se sia vero che l'ente si divide in positivo e negativo; che l'ente positivo si divide in reale e di ragione; che l'ente reale si divide in creato e increato. A questa domanda egli risponde che tutte e tre le divisioni sono abusive: non vi è un concetto comune, sia esso univoco o analogo, all'ente positivo e negativo, reale e di ragione, creato e increato. Ora, a proposito delle prime due divisioni Pasqualigo sostiene una tesi condivisa da molti altri autori. Degno di nota, invece, è il fatto che il teatino veronese rifiuti la divisione dell'ente reale in ente increato ed ente creato. La ragione di tale tesi è fondamentalmente una sola: Pasqualigo ritiene che la creatura sia ente in virtù della propria ratio di ente e che, quindi, sia ente intrinsecamente e assolutamente. Ora, tale ratio non può essere identica in Dio e nella creatura: essa è intrinseca a Dio e alla creatura; pertanto, nel primo è increata, nella seconda è creata. Ma non può neppure essere analoga. Pasqualigo nega che abbia senso parlare di analogia di attribuzione intrinseca. Attribuzione e intrinsecità, egli scrive, si oppongono. Si deve piuttosto dire, prosegue, che quando la ratio analoga è intrinsecamente presente in tutti gli analogati, si ha analogia di proporzione; quando la ratio analoga è intrinsecamente presente solo in uno degli analogati, si ha analogia di attribuzione. Ma se così è, la creatura certamente non è ente per analogia di attribuzione. Non può però essere 'ente' neppure per analogia di proporzione: la creatura, come si è detto, è ente per la ratio entis propria, non per l'altrui. Ne viene che Dio non è ente; propriamente, egli è qualcosa di superiore all'ente. Certamente Dio è reale ed esistente, spiega il nostro autore, ma lo è grazie a una ratio superiore a quella dell'ente. Detto questo, Pasqualigo precisa che egli non nega che vi sia proporzione tra le funzioni che la ratio entis svolge nella creatura e quelle che la ratio superiore all'ente svolge in Dio. Né egli nega che l'ente della creatura dipenda da Dio. E neppure nega che sia possibile attribuire a Dio la ratio dell'ente. Tuttavia, aggiunge, attribuire tale ratio a Dio è attribuire a Dio un nome tratto dalle creature per indicare in lui la capacità di causare l'ente delle creature; si tratta dunque di una predicazione per un'analogia di attribuzione che non solo è estrinseca, ma ha il suo analogato principale non in Dio, bensì nella creatura. ${ }^{22}$

Di Vona riassume il senso della dottrina di Pasqualigo osservando che l'ambito della metafisica è per il teatino veronese l'ente naturale.

22 DI VONA. 1984, p. 210-17. ID. 1994, p. 57-8, note 8 e 11. Di Vona ha preso in esame i gruppi di disputationes, dalla 26 alla 32 e dalla 33 alla 42, riunite sotto i titoli 'De divisione entis' e 'De conceptu entis'. 
Così facendo, Pasqualigo in primo luogo rende la metafisica totalmente indipendente dalla teologia, presa non solamente nel senso di 'teologia soprannaturale', ma anche in quello di 'teologia razionale'. In secondo luogo fa della metafisica un'ontologia pura, la quale ha per suo oggetto solamente la sostanza e l'accidente materiali. ${ }^{23}$

Per quanto sono capace di comprendere, ritengo che l'interpretazione di Di Vona sia in buona parte condivisibile; mi sembrano tuttavia necessarie due puntualizzazioni. Innanzi tutto osservo che Pasqualigo non elimina dalla sua metafisica ogni riferimento all'ambito teologico: anche per lui, infatti, l'ente deriva da Dio. Da questo punto di vista, egli non appare distante da quanto sosteneva Tommaso d'Aquino: lo ens communis, che per l'Aquinate è soggetto della metafisica, è causato da Dio. Si potrebbe forse interpretare la posizione di Pasqualigo come un tentativo di rigorizzare la confusa posizione dell'Aquinate. Secondo Tommaso, l'ente comune a sostanza e accidente materiali ha due caratteristiche: da un lato è causato da Dio; dall'altro è una nozione analogica della quale l'ente detto di Dio è l'analogato principale. Ebbene, mi sembra che questa posizione contenga un equivoco: confonde la ratio analoga (l'ente trascendentale, che non ha causa) con la ratio dell'analogato secondario (l'ente creato, che per Tommaso è causato). Mi sembra anche che questo equivoco costituisca la ragione di fondo per la quale tutti gli interpreti dell'Aquinate hanno dovuto faticare non poco e normalmente senza successo - per fornire una ricostruzione unitaria del pensiero del dottore domenicano circa la natura della metafisica. Al contrario, per Pasqualigo l'ente comune a sostanza e accidente materiali è una nozione univoca. Essa diviene analogica solamente allorché è utilizzata per parlare di Dio; nel qual caso l'ente detto di Dio è analogato secondario. Stabilito questo punto, la tesi secondo la quale tale ente è causato da Dio può essere affermata con minore difficoltà. Chiarito tutto questo, resta che condivido un aspetto essenziale delle considerazioni formulate da Di Vona: benché per Pasqualigo l'ente materiale derivi da Dio, questa derivazione non svolge alcun ruolo nell'indagine metafisica su tale ente.

Un secondo punto che richiede una puntualizzazione concerne l'uso, al fine di descrivere la posizione di Pasqualigo, dell'espressione 'ontologia pura'. Per fare luce su questo tema, può risultare utile un confronto con un aspetto della posizione di Suárez. La metafisica del Granadino è tutt'altro che un'ontologia pura; probabilmente, non si può neppure dire che essa sia un'ontologia. Le ragioni che escludono la possibilità di descrivere la metafisica di Suárez come un'ontologia sono più d'una.

${ }^{23}$ DI VONA. 1994, p. 54-55. 
Tra queste, quella per cui il pensatore gesuita fa propria una dottrina che Antonio Trombetta aveva sviluppato a partire da un passo del libro sesto della Metafisica di Aristotele. Qui si legge che se non esistessero enti spirituali, la fisica sarebbe la filosofia prima. Il senso che Trombetta e Suárez danno a questa affermazione è il seguente. Se non esistessero enti immateriali, la nozione di 'ente reale' sarebbe necessariamente connessa alla nozione di 'materia'. Dunque, l'astrazione da parte della mente della nozione di 'ente prescindente dalla materia' dalla nozione di 'ente materiale' sarebbe certamente un atto possibile, tuttavia sarebbe un atto arbitrario. Detto in altri termini, la nozione di 'ente prescindente dalla materia' sarebbe non una nozione 'reale', bensì una nozione 'di ragione'; sarebbe una nozione alla quale non corrisponde nulla nella realtà. ${ }^{24}$

Ora, la posizione di Pasqualigo appare un diretto rovesciamento di questo argomento. Egli ritiene che non possa esservi una nozione di 'ente' comune a ciò che è materiale e a ciò che è spirituale per una ragione uguale e contraria a quella fatta valere da Suárez: la nozione di ente di cui si occupa la metafisica è una nozione 'reale'; ma essa è tale solamente se essa non esclude la possibilità di darsi nella materia. Ne viene che, secondo il nostro autore, la nozione di ente indifferente a ciò che è materiale e a ciò che è spirituale implica una contraddizione: dovrebbe infatti essere tale sia da non escludere la possibilità di darsi nella materia, sia da escludere tale possibilità. È tale nozione, pertanto, ad essere arbitraria. Questo comporta che - nella prospettiva di Pasqualigo - la metafisica sia una scienza possibile solamente se l'ente di cui essa si occupa è astratto dall'ente materiale unicamente in forza di un'operazione dell'intelletto. In altri termini: secondo Pasqualigo, la metafisica è possibile precisamente perché non si può parlare di 'enti spirituali' ed è distinta dalla fisica solamente in forza dell'opera dell'intelletto.

Si può dunque dire che la metafisica di Pasqualigo sia un'ontologia pura? Una ragione di dubbio sta nel fatto che, per il nostro autore, l'oggetto adeguato di tale scienza non è l'ente trascendentale. Ciononostante, si può forse rispondere affermativamente alla domanda ora posta: solo la metafisica, secondo il Veronese, si occupa dell'ente come tale; inoltre, essa non si occupa per nulla di ciò che è spirituale. Ammesso che si possa denominare tale scienza 'ontologia', dunque, saremmo di fronte a un'ontologia più 'pura' addirittura di quella, ad esempio, di Francesco di Appignano. Quest'ultimo infatti, pur distinguendo metaphysica generalis

${ }^{24}$ Per questo aspetto della dottrina di Suárez, mi permetto di rinviare ai saggi che ho già menzionato nella nota 1. Per quanto riguarda la posizione di Trombetta, mi permetto di rinviare a FORLIVESI. 2008. 
e metaphysica particularis, assegnava alla prima, e non alla seconda, il compito di dimostrare l'esistenza di Dio. Resta che la metafisica di Pasqualigo non ha nulla a che vedere con le 'ontologie pure' di ambiente calvinista o illuminista. Essa non si occupa di rationes generalissimae che prescindano dalla materia o dalla finitudine a tal punto da potersi dare anche in enti spirituali o in Dio. L'ente di cui la metafisica pasqualighiana si occupa non si contrae all'ente infinito e all'ente finito, e neppure all'ente materiale e all'ente spirituale; pertanto questa metafisica è una scienza 'superiore' alla fisica e alla matematica, ma non alla teologia razionale o alla pneumatologia. È un'ontologia dell'ente materiale considerato, per mezzo della sola opera della mente, a prescindere dalla sua intrinseca e necessaria materialità.

\section{Referências}

DI VONA, P. I concetti trascendenti in Sebastiàn Izquierdo e nella scolastica del Seicento. Napoli: Loffredo, 1994.

. "La sovreminenza di Dio nella scolastica del '600". In: La storia della filosofia come sapere critico. Studi offerti a Mario dal Pra. Milano: Franco Angeli, 1984, p. 210-217.

FORLIVESI, M. "Approaching the Debate on the Subject of Metaphysics Between Later Middle Ages and Early Modern Age: The Ancient and Medieval Antecedents", Medioevo, 34 (2009a), in corso di stampa.

. (ed.). Antonio Bernardi della Mirandola (1502-1565). Un aristotelico umanista alla corte dei Farnese. Firenze: Leo S. Olschki, 2009b.

“"Quae in hac quaestione tradit Doctor videntur humanum ingenium superare'.

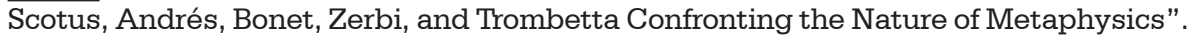
Quaestio 8 (2008), in corso di stampa.

. "Impure Ontology. The Nature of Metaphysics and Its Object in Francisco Suárez's Texts", Quaestio, 5 (2005), p. 559-586.

. Ontologia impura. La natura della metafisica secondo Francisco Suárez. 2004a.

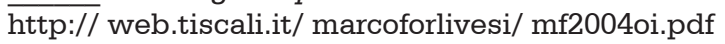

. "Ontologia impura. La natura della metafisica secondo Francisco Suárez". In: Francisco Suárez. "Der ist der Mann". Homenaje al prof. Salvador Castellote. Valencia: Facultad de Teología "San Vicente Ferrer", 2004b, p. 161-207.

. L'ontologia di Bartolomeo Mastri nelle 'Disputationes in XII libros Metaphysicorum'. Diss. Università Cattolica di Milano 1999 (liberamente accessibile all'indirizzo internet http:// web.tiscali.it/ marcoforlivesi/ mf1999om.pdf).

LAMANNA, M. “'De eo enim metaphysicus agit logice'. Un confronto tra Pererius e Goclenius". Medioevo, 34 (2009), in corso di stampa.

PORRO, P. “Introduzione. Dalla 'Metafisica' alla metafisica, e ritorno: una storia medievale", Quaestio, 5 (2005), p. IX-LI.

POZZO, R. "Cornelius Martini sull'oggetto della metafisica". Medioevo, 34 (2009), in corso di stampa. 
RUFFINENGO, P. P. "L'oggetto della metafisica nella scuola tomista tra tardo medioevo ed età moderna", Medioevo, 34 (2009), in corso di stampa.

VEZZOSI, A. F. I scrittori de' Chierici regolari detti Teatini. Roma: Nella stamperia della sacra Congregazione di Propaganda fide, 1780, vol. 2.

ZACHARIA Pasqualigus. Sacra speculativa doctrina de Deo coeterisque divinitus revelatis ex theologicis principijs ad scholasticae lecturae methodum deducta. Venetiis: Apud Bertanos, 1650.

. Disputationes metaphysicae. 2 voll.: vol. 1, Romae: Ex typographia Andreae Phaei, 1634; vol. 2, Romae: Typis Francisci Caballi, 1636. 